\title{
KLAUS JACOBI
}

,Gut' und ,schlecht’

Die Analyse ihrer Entgegensetzung bei Aristoteles, bei einigen Aristoteles-Kommentatoren und bei Thomas von Aquin 


\title{
,GUT' UND ,SCHLECHT* \\ DIE ANALYSE IHRER ENTGEGENSETZUNG BEI ARISTOTELES, BEI EINIGEN ARISTOTELES-KOMMENTATOREN UND BEI THOMAS VON AQUIN
}

\author{
von KLaus JacoBı
}

Problemstellung

\begin{abstract}
,Gut' und, schlecht' als Beispiele für konträr Entgegengesetztes
(Aristoteles, Cat.)
\end{abstract}

In Aristoteles' Untersuchungen über die Weisen, wie etwas zu etwas in Gegensatz stehen kann', gilt das Begriffspaar ,gut - schlecht' als einleuchtendes Beispiel für den konträren Gegensatz ${ }^{2}$. Genauer wird der Gegensatz zwischen dem Guten und dem Schlechten demjenigen Typus von Kontrarietät zugeordnet, bei welchem es etwas Mittleres zwischen den Extremen gibt; nicht notwendig kommt einem Subjekt, dem diese konträren Prädikate zukommen können, auch tatsächlich das eine oder das andere dieser Prädikate $\mathbf{z u}^{3}$.

Für ,gut' und ,schlecht' können in diesen Analysen auch dic Worte

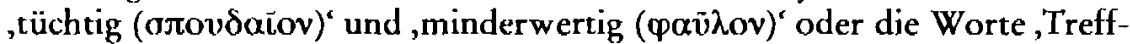

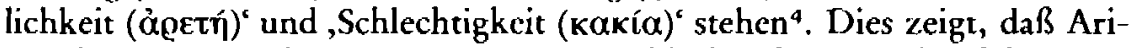
stoteles, wenn er den Gegensatz, gut - schlecht' als Beispiel anführt, von einer Verwendung ausgeht, in der diese Prädikate primär - wenn auch vielleicht nicht ausschließlich - von menschlichen Handlungen ausgesagt werden. Der Handelnde selbst gilt als gut (bzw. als schlecht), sofern sein Handeln und scine Intentionen habituell gut (bz.w. schlecht) sind. ,Gut' und ,schlecht' scheinen demnach Bestimmungen in der Kategorie des Beschaffenseins $z$ scin.

1 Aristotcles, Cat. 10; vgl. Top. II 8; Meraph. $\Delta 10 ; 14$.

2 Cat. 10,11 b $19-21$; vgl. 11 b $35-36 ; 12$ a 9-25; 11,13 b $36-14$ a $6 ; 14$ a $19-25$; ferner $7,6 \mathrm{~b} 15-16$.

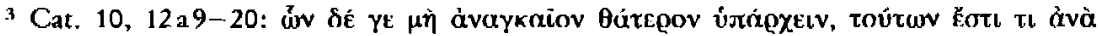

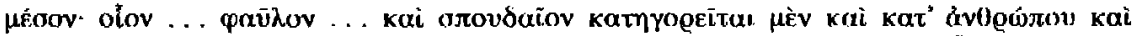

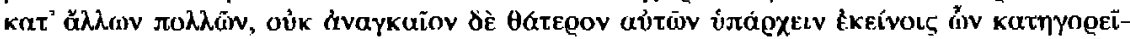

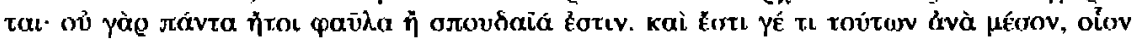

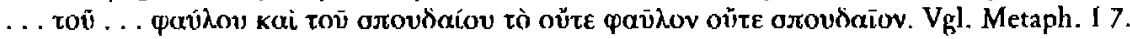

4 Cat. 7,6 b $15-16 ; 11,14$ a $22-25$. 


\section{,Gut' als homonymes, überkategoriales Prädikat}

(Aristoteles, Top.; EN)

Nun betont Aristoteles an anderen Stellen, daß die Bestimmungen, gut ${ }^{*}$ und ,schlecht" verschiedenen Kategorien zugehören können. Gut (bzw. schlecht), so wird in der "Topik“ ausgeführt, kann etwas nicht nur als ein irgendwie Beschaffenes genannt werden, sondern auch, sofern es eine gute (bzw. schlechte) Beschaffenheit bewirkt, ferner als Zeitpunkt oder auch als ein Wieviel. ,Gut' und, schlccht' sind folglich homonym ${ }^{5}$. In der „Nikomachischen Ethik" geht Aristoreles noch cinen Schritt weiter. Nicht nur in verschiedencn, sondern ,in allen Kategorien“ wird ,gut" ausgesagt, also ,in gleich viel Bedcutungen wic , seiend" "6. Die Frage nach dem Zusammenhang aller Bcdeutungen von, gut ' wird aufgeworfen ${ }^{7}$, doch geht Aristoteles dieser Frage weder hier noch an irgend einer anderen Stelle nach.

Ein Teilproblem, viclleicht sogar ein Schlüsselproblem bezüglich der von Aristoteles unbeantwortet gelassenen Frage besteht darin, zu prüfen, ob ,gut' in allen Bedcutungsformen und Verwendungsweisen einen Gegenbcgriff , schlccht' zuläßt und ob das logische Verhältnis $z$ wischen ,gut ${ }^{\prime}$ und ,schlecht' stets das der Kontrarictät ist ${ }^{8}$. Aristoteles scheint dies vorauszusetzen. Auch an Stellen, an denen er hervorhebt, daß, gut' nicht in irgendeiner Gattung ist, sctzt er ,schlecht' als konträren Gegenbegriff, von welchem dann ebenfalls gelten soll, daß er nicht in irgendeiner Gattung ist ${ }^{9}$. Ob diese Voraussetzung richtig ist, ist z.weifelhaft. Zumindest der Substanz (oủoí $\alpha$ ), so sagt Aristoteles, ist nichts konträr ${ }^{10}$, und ,ovơí $\alpha^{\prime}$ ist diejenige Bedeutung von ,seiend', auf die alle anderen Bedeutungen bezogen sind. Wenn nun ,gut' in gleich viel Bedeutungen wie ,seicnd' ausgesagt wird, muß es dann nicht zumindest eine Bedeutung geben, in welcher auch ,gut" "nichts konträr" ist?

${ }^{5}$ Aristoteles, Top. I 15, 107a 3-12. Vgl. J. L. Ackrill, Aristotle on 'Good' and the Categories, in: Islamic ['hilosophy and the Classical Tradition, hg. S. M. Stern, A. Hourani, V. Brown, Oxford 1972, 17-25; Nachdruck in: Articles on Aristotle, 2. Ethics and Politics, hg. J. Barnes, M. Schofield, R. Sorabji, London 1977, 17--24.

- Aristoteles, EN I 4, 1096 a 23-29, zitiert: a 28-29 und a 23-24; vgl. a 17-23.

7 EN I 4, 1096b 26-31.

" Fragetl solcher Art spielen auch in modernen sprachanalytischen Untersuchungen eine großc Rulle; vgl. G. H. von Wright, The Varictics of Goodncss, London-New York 1963.

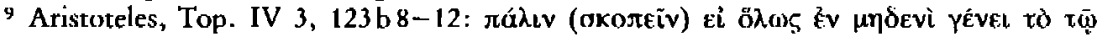

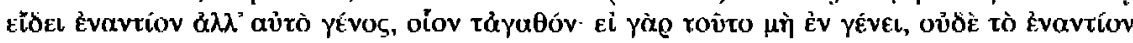

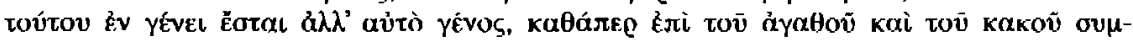

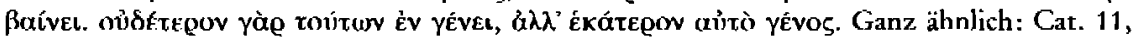
14 a 19-25, zitiert unten S. 31 .

10 Cat. 5, 3b 24-27; vgl. Mctaph. N 1, 1087b 2- 3. 


\section{Verschiedenbeit, Unterschied, Kontrarietät, Haben - Beraubung (Aristoteles, Metaph.)}

Wenn man der gestellten Frage auf aristotelischer Grundlage nachgehen will, dann sind, obwohl rom Gegensatz, gut - schlecht' nicht thematisch gehandelt wird, dic Untersuchungen der "Metaphysik" mit in Betracht z.u ziehen. Die Wissenschaft vom ,,Seienden als Seienden" und von dem, „,was diesem an sich zukommt"11, hat nicht nur die Bedeutungen von ,seiend" $\mathrm{zu}$ analysieren und deren Einheitspunkt aufzuweisen ${ }^{12}$, sondern auch dic Negationsformen von, seiend ${ }^{4} \mathrm{zu}$ untersuchen ${ }^{13}$. Ebenso hat sie auch hinsichtlich der mit , seiend' konvertiblen Prädikate ${ }^{14}$ deren Bedeutungsund deren Negationsformen zu klären. Mit ,seiend' konvertibel ist, eines'. Zur Theorie des Prädikats, eines` und seiner Negationsformen gehört die Theorie von Identität und Verschiedenheit; zu dieser Theorie wiederum gehört die Bestimmung dessen, was ,Unterschied ( $\delta\llcorner\alpha \varphi \circ \varrho \alpha ́)$ ' besagt. Von diesem Punkt ausgehend muß auch geklärt werden, was ,Kontrarietät als Sonderart von ,Unterschied ${ }^{`}$ besagt und unter welchen Bedingungen Prädikate zueinander konträr sind ${ }^{15}$.

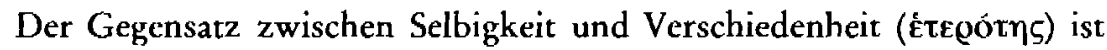
kein kontradiktorischer Gegensatz. Während nämlich bei kontradiktorisch Entgegengesetztem ohne jede Einschränkung immer je das eine Glied des Gegensatzes wahr und das andere falsch sein muß, gilt für dic Gegensatzglieder, dasselbe - das anderec eine Anwendungsbedingung: Nur unter der Voraussetzung, daß nur von Seiendem, nicht von Nicht-Seiendern die Rede ist, gilt der Satz. Ein jedes (nämlich: Seiende) ist im Verhältnis zu jedem (Seienden) entweder dasselbe oder cin anderes" ${ }^{\text {16. }}$.

Unterschied ( $\delta\llcorner\alpha \varphi 0 \varrho \alpha ́)$ ist gegenüber Verschiedenheit der engere Begriff. Wenn unterschieden wird, wird nicht nur vorausgesetzt, daß beide Unterschiedene seiend sind, sondern darüber hinaus auch, daß sie irgendeine inhaltliche Bestimmung miteinander gemein haben. Die Unterscheidung besteht in der Angabe einer weiteren Bestimmung, durch wclche das Unterschiedene sich unterscheidet ${ }^{17}$. Kontrarietät ( endete Unterschiedenheit ${ }^{18}$. Eine gattungshafte Gemeinsamkeit bleibt vorausgesetzt. Innerhalb dieser Gattung kann es zwar in der für die Unterscheidung relevanten Hinsicht viele Gradabstufungen geben, aber nur einen

\footnotetext{
11 Aristoteles, Metaph. Г 1, 1003 a 21-22.

12 Metaph, Г 2, 1003 a 33.

13 Metaph. $\Gamma 2,1003$ b $7-10$.

14 Metaph. Г 2, 1003 b 22-24.

is Metaph. I 2, 1003 b $33-1004$ a 21.

It Metaph. I 3, 1054 b 18-22; vgl. Г 7, 1011 b 23-28; Cat. 10, 13 b 27-35.

17 Metaph. I 3, 1054 b 22-31; vgl. $\triangle 9,1018$ a 12-15; Cat. 11, 14 a 15-19.

18 Metaph. I 4, 1055 a 3-16.
} 
größten Unterschicd. Also ist, wenn dieselbe Hinsicht gewahrt bleibt, je einem immer nur eines konträr ${ }^{14}$.

Aristoteles führt den konträren Gegensat\% auf den Gegensatz von Haben

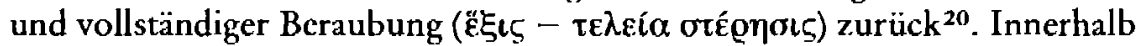
einer gemeinsamen Gattung besteht dic größte Differenz darin, daß die differenzierende Bestimmung bei dem einen der Unterschiedenen voll ausgebildet vorhanden ist, bei dem anderen dagegen gänzlich fehlt. Diese Rückführungsthese bedarf jedoch der Ergänzung. Unbeachtet bleibt in ihr, in welchem Verhältnis die differenzierende Bestimmung zur gemeinsamen Bestimmung steht. Im wichtigsten Fall der Unterscheidung, nämlich im Fall der Spezifikation, tritt dic Differenz nicht gleichsam additiv zur Gattungsbestimmung hinzu, sondern sie differenziert die Gattungsbestimmung selbst. Wenn $x$. B. der Artbegriff ,Mensch“ als, vernunftbegabtes Sinnenwesen' definiert wird, dann ist damit gemeint, daß das Sinnenwesensein als solches im Menschen durch Vernunftbegabung geformt und bestimmt ist. Nun gibt es kein Wesen, dem bloß die Bestimmung, Sinnenwesen“ und kcine weitere spezifizierende Bestimmung zukäme. Das Sinnenwesenscin ist in jc anders gearteten Sinnenwesen je unterschicdlich geformt und bestimmt, nirgends aber einfach unbestimmt. Wenn etwa definiert werden sollte, was cin Pferd ist, so wäre klarzustellen, daß auch mit der bloß negativen Bezeichnung, nicht-vernunftbegabt' cin spezifizierender (und weiter differenzicrbarer) Sinngehalt gemeint ist ${ }^{21}$. ,Rückführung der Kontrarietät auf den Gegensatz von Haben und vollständiger Beraubung kann also nicht heißen, daß das Kontrarietätsverhältnis mit dem Verhältnis "Haben - Beraubung" gleichgesetzt werden dürfte. Die „Rückführung" crklärt das Moment der Entgegengesetztheit der contraria; die Betrachtung der artbildenden Unterschiede lehrt, daß - zumindest in diesem Fall beide contraria einen positiven Sinngehalt bezeichnen ${ }^{22}$, während die bloßc Beraubung ,nichts setzt" ${ }^{\prime 23}$.

Der Gegensatz ,gut - schlecht' als Gegensatz von Haben und Beraubung (Johannes Philoponos)

Wenn man mit den hicr in Kürzc erinnerten begrifflichen Unterscheidungen $z$ wischen Verschiedenheits- und Gegensatztypen an den Gegensatz

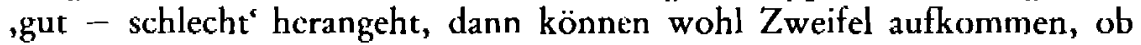

\footnotetext{
19 Metaph. I 4, 1055a 19-23.

20 Metaph. I 4, 1055a 33-35; b 14-15; b26-29; vgl. Г 2, 1004 a 12-16; 6, 1011 b $16-20$.

21 Mctaph. I 8, 1057b 35-1058 a 8; a 21-28.

22 Vgl. Thomas von Aquin, In Metaph. L. X I. IV, n. 1988; I. VI, nn. 2037-2038; L. IV I. XV, n. 719.

$23 \mathrm{Vgl}$. Thomas von Aquin, In Mctaph. I.. X l. VI, лn. 2043-2045.
} 
Aristoteles' Bestimmung dieses Gegensatzes überhaupt das Rechte trifft. Bei einigen der spätantiken Aristoteles-Kommentatoren jedenfalls sind solche Zwcifel deutlich. Diese Kommentatoren sind aus der neuplatonischen Schule hervorgegangen, deren besonderes Interesse für Fragen nach Einheit und Vielheit und nach Identität und Differenz bekannt ist. Wic stellt sich ihnen Aristoteles' These, ,gut' und, schlecht' seien cinander konträr, dar?

Johannes Philoponos hält diesc These für ungenau; nach seinem Verbesserungsvorschlag muß hier der Gegensatz von Haben und Beraubung angesetzt werden. Zur Begründung sciner Gegenthese rekurriert er auf die Lehre, daß bci konträren Gcgensätzen - z. B. ,weiß - schwarz ${ }^{c}$ - jedes Gegensatzglied ,einen bestimmten Sinngehalt hat" und deshalb ,eine Art bildet". ,Schlecht" aber bezeichne bloße ,Ungemessenheit" und könne deshalb nicht spezifizieren. Philoponos vertritt diese Gegenthese generell für jede Verwendung von, schlecht'. Auch ,Schlechtigkeit' als Gegenbegriff zu "Trefflichkeit' bezeichnc nur ,Abwescnheit von Trefflichkeit", also kein konträr Entgegengesetztes, sondern bloße Beraubung ${ }^{24}$.

Philoponos ist weiter der Auffassung, daß ,gut' nicht in jeder Verwendung cinen Gegenbegriff zuläßr. Mit dem Hinweis auf den Satz aus der Kategoricnschrift des Aristoteles, der Substanz sei nichts konträr, begründet dieser Kommentator seine These, das ,an sich Gute, das substanzhaft Gott zugrundeliege“, habe kein konträr Entgegengesctztes; nur das ,,bci-

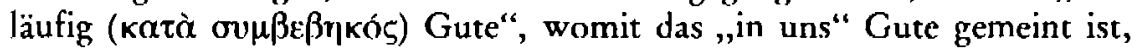
werde in Entgegensetzung ( nannt ${ }^{25}$.

\section{,Gut' und ,schlecht' als gänzlich voneinander geschieden? (Simplikios gegen Plotin)}

Der Stil, in dem der scharfsinnige und gelehrte Simplikios dic „Kategoricn" kommentiert, gibt der kritischen Diskussion großen Raum. Simplikios prüft Thesen und Argumentationen, nicht nur die von Aristoteles selbst stammenden, sondern auch die des ,großen Plotin“"26 und seiner

24 Philoponi (olim Amonii) In Aristotelis Categorias Commentarium, ed. $\Lambda$. Busse, Berlin $1898,190,28$ - 191,3 zu: Cat. 11, 14a 19-21 (= CAG XIII, 1).

25 Philoponos, In Cat, 170,31-171,4 zu: Cat. 10, 11 b21.

2h Simplikios zitiert Plotin häufiy mil diesem Beinamen; er benennt ihn auch als ,den götrlichsten". Vgl. die in den Namenregistern der kritischen liditionen angegebenen Belegstellen. Der Kommentar des Simplikios zur Kategoricnschrift wird im folgenden nach der griechischen und nach der lateinischen Fidition ziticrt: Simplicii In Aristotelis Categorias Commentarium, ed. C. Kalbfleisch, Berlin 1907 (= CAG VIII) - Simplicius, Commentaire sur les Catégories d'Aristote, Traduction de Guillaume de Moerbeke, ed. A. Pattin (tome I en collaboration avec W. Stuyven), Louvain - Paris 1971-1975 (= Corpus Latinum Commentariorum in Aristotelem Craccorum V, 1-2). 
Schüler wie auch die der Stoiker. Einige der von ihm geführten Diskussionen betreffen die Frage, wie, gut' und, schlecht' einander entgegengesetzt sind. Sie können helfen, die in dieser Frage liegenden Probleme scharf zu sehen.

Plotin hatte in sciner Abhandlung ,Was das Schlechte ist und woher es stammt" 27 das Schlechte als "Abwesenheit" 28 und „Beraubung“29 von jeglichem Guten bestimmt. Was das Gute ist, umschrcibt Plotin mit Titcln wie „das Unbedürftige" ${ }^{\text {30 }}$, „Maß und Grenze aller Dinge" Einheit von allem" 32 , ,erste Wirklichkeit" und „erste Wesenheit" 33 . Dem Guten stellt er das Schlechte als „Ungemessenheit", als ,, das Unbegrenzte“, „Ungestaltete“, „Bedürftigc“" und als „Armut" gegenüber ${ }^{34}$; all dies sind

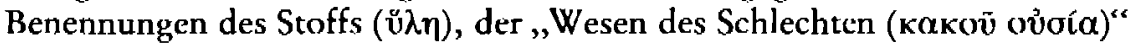
sei $^{35}$.

Anknüpfend an Platon ${ }^{36}$ und in Gegenwendung gegen Aristoteles' Ausspruch, der Substanz sei nichts konträr ${ }^{37}$, fragt Plotin nun, wie es überhaupt zu dem Guten einen Gegensatz (Ěvoviíov) geben könne, wenn doch „das Gutc Wesenheit (oủoía) ist" oder gar noch ,jenseits der Wesenheit liegt" "38. Seinc Antwort lautet: Der Gegensatz zwischen dem Guten und dem Schlechten sei freilich kein Gcgensatz im gewöhnlichen Sinn; es gebe hicr überhaupt keine Gemeinsamkeit, auch nicht im Sinne einer gattungshaften Einheit, sondern von allem, was das eine brauche, um sein Wassein

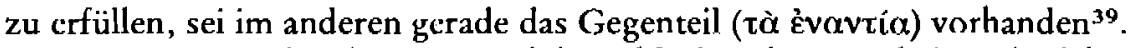
Entgegengesetzt seien das Gute und das Schlechtc als Wesenheit und NichtWesenheit (ov̉oí $\alpha-\mu \grave{\eta}$ ov̉oí $\alpha)^{40}$, wic lichthaftes Sein und Schatten ${ }^{41}$, entgegengesetzt im Sinne von "gänzlich voneinander geschieden" 42 und „den weitesten Abstand voneinander babend"43. Es handelt sich hier also

27 Plotin, Enn. I 8. Potins Schriften, übers. v. R. Harder, Ncubcarbeitung mit gricch. I.esetext u. Anm. fortgeführt v. R. Beutler u. W. Theiler, I3d. V, Hamburg 1960.

24 Finn. I 8, 1, 11-12.

29 Enn. I 8, 1, 17; vgl. I 8, 11, 1-4.

3o Enn. I 8, 2, 4.

31 Enn. I 8, 2, 5; vgl. I 8, 6, 41-44.

32 Enn. I 8, 2, 7--21.

33 Enn. $18,2,21-22$.

34 Enn. I 8, 3, 12-20; vgl. I 8, 6, 41-44; I 8, 8, 37-38.

35 Enn. I 8, 3, 35-40; vgl. I 8, 9, 15-18.

36 Platon, Theait. 176a; Plotin, Enn. I 8, 6, 20-25.

37 Aristoteles" Name wird von Plotin nicht genannt. Fs ist aber klar, daß er sich auf Cat. 5, 3b 24-27 bexieht; Simplikios beurteilt mit Kecht in scinem Kommentar zu dieser Passage der Kategorienschrift Plotins Ausführungen als Gegenthese gegen Aristotcles.

38 Plotin, Finn. I 8, 6, 27.

39 Enn. I 8, 6, 35-38.

40 Enn. $18,6,32$.

41 F.nn. I 8, 3, $1-9$.

42 Enn. I 8, 6, 38; I 8, 6, 54-56.

43 Enn. I 8, 6, 38. 
nach Plotin nicht um eine äußcrste Differenz innerhalb einer gemcinsamen Gattung, sondern um den Urgegensatz. zwischen der überwesenhaften Einheit, die Prinzip alles Seienden ist, und dem Alleräußersten, das aus ihm hervorgeht ${ }^{44}$, dem gan\% formlosen ${ }^{45}$ und qualizätslosen Stoff ${ }^{46}$, der reine Beraubung ist ${ }^{47}$.

Simplikios zeigt die Schwicrigkeiten, die in der Theorie Plotins und „aller, die aus der Einheit anfangend" philosophieren ${ }^{48}$, liegen. Sofern doch auch das dem Gutcn Entgegengesetzte aus dem Guten stammt, so lautet Simplikios' in verschiedenen Varianten vorgctragener Einwand, muß es auch mit diesem etwas gemcin haben und ihm in irgendeiner Weise zugeordnet bleiben. Es kann also zwischen dem Guten und dem Schlechten nicht die von Plotin behauptete völlige Disparatheit bestehen ${ }^{49}$. Und wenn das „Äußerste“ seine ganze Existenz aus dem Prinzip hat, wie kann es diesem übcrhaupt entgegengesetzt $\operatorname{sein}^{50}$ ? Disparat könnte allenfalls ein dem Guten gleichrangiges Gegenprinzip sein; dann aber wäre zum einen unverständlich, inwiefern das cine Prinzip „das Gute“, das andere aber "das Schlechte“ sein sollte, zum anderen, wic diese Prinzipien übcrhaupt zusammenwirken können ${ }^{51}$.

Schwierigkeiten in Aristoteles' Behandlung des Gegensatzes, gut - schlecht': Sind die Kontrarietäts- und die Homonymietbese miteinander verträglich? (Simplikios)

Der Kommentator Simplikios findet aber auch in Aristoteles' Ausführungen über den Gegensatz, gut - schlecht' erhebliche Schwierigkeiten. Lehrreich ist besonders die ausführliche und gründliche Diskussion der bereits erwähnten Passage aus c. 11 der Kategorienschrift. Dort heißt es:

„Notwendig ist jedes Konträre entweder in derselben Gattung, oder (es ist) in konträren Gattungen, oder dic Glieder des konträren Gegensatzes sind selbst Gattungen. Weiß und schwarz nämlich sind in derselben Gattung - ihre Gattung ist: Iarbe -, Gerechtigkeit und Ungerechtigkeit dagegen sind in konträren Gattungen - für das eine ist die Gattung: Trefflichkeit, für das andere: Schlechtigkcit -, gut und schlecht schließlich sind nicht in einer Gattung, sondern für sie trifft $z u$, daß sie Gatrungen für anderes sind." 52

44 Enn. 18, 7, 16-20.

45 Finn. $18,9,15-18$

46 Enn. I 8, 10.

47 Enn. I 8, 11, 1-4.

44 Simplicius, In Cat., CAG 109,29-32 - CLCAG 147 - 148, Zeilen 21-23.

49. In Cat., CAG 109,7-11 - CI.CAG 147, 95-00; CAG 110,6-11- CLCAG 148, $30-35$.

50 In Cat., CAG 109,12-17 -. CLCAG 147,2-7.

51 In Cat., CAG 109, 20-29 - C.LCAG 147, 10-21.

52 Aristoteles, Cat. 11, 14 a 19- 25. 
Strittig ist das dritte Glied dicser Unterscheidung. Simplikios blickt auf eine lange Kontroverse zurück, die keine befriedigende Lösung gebracht hat. Er zitiert Stellungnahmen zu dieser Passage von Nikostratos ${ }^{53}$, von Philosophen aus dem Kreis um Porphyrios ${ }^{54}$, von Theophrast ${ }^{55}$ und von Iamblichos $^{56}$, und er zieht crgänzend cine Passage aus der Schrift des Archytas „Uber die Gegensätze" hinzu" ${ }^{57}$, in der er die Vorlage des Aristoteles sieht.

Die These, es gebe mindestens cinen Fall von Kontrarietät, nämlich den Gegensatz, gut - schlecht', ohne daß es für die Gegensatzglieder eine gemeinsame Gattungsbestimmung gebe, stcht in Konflikt mit der Begriffsbestimmung von Kontrarietät, in der cine gemeinsame Gattung gerade gefordert ist ${ }^{54}$. Außerdem ist nicht klar, ob die genannte These mit der Kategorienlehre vereinbar ist. Die Kategorien sind höchste Gattungen. Will Aristoteles sagen, die Bestimmungen ,gut' und, schlecht ${ }^{\star}$ fielen nicht mehr unter eine Kategorie, weder unter dic des Beschaffenseins, noch unter eine andere? Dann hätte er nicht hinzufügen dürfen, ,gut' und ,schlecht' seien selbst Gattungen; diese Hinzufügung bringt ihn in Konflikt mit der Kategorienlehres9.

Es liegt nahe, eine abschwächende Interpretation der These zu versuchen. Danach will Aristoteles gar nicht bestreiten, daß, gut' und ,schlecht' Bestimmungen innerhalb der Kategorie der Beschaffenheit sind; mit dem Wort ,Gattung' in der zitierten Passage sind dic Kategorien nicht mitgemeint. Es kommt ihm vielmehr auf den Nachwcis an, daß konträre Gegensätze einc unterschiedliche Stellung in einer Begriffshicrarchie einnehmen können. Erstens können contraria als Arten unmittelbar einer gemeinsamen nächsten Gattung zugeordnct sein. Zwcitens können contraria als Arten unmittclbar Gattungen zugeordnet sein, die ihrerseits konträr sind; gemein-

53 Simplicius, In Cat., CAG 414,26-33 - CLCAG 569,8-15; vgl. CAG 388,4-13 CLCAG 529, 57-67.

54 In Cat., CAG 414,33-415,7 - CLCAG 569-570, Zcilen 15-24.

55 In Cat., CAG 415,15-19- CLCAG 570, 32-37.

56 In Cat., CAG 415,20-22 - CLCAG 570,37-40; CAG 415,30-34 - CLCAG 570-571, Zeilen 49--52 (ungenau überserzt).

57 In Cat., CAG 416,7-20 - CLCAG 571,63-76.

${ }^{58}$ In Cat., CAG 414,27-30 - CI.CAG 569,8-12; CAG 415,34-416,2 - CLCAG 571,52-57. Die 'latsache, daß die genannte Begriffsbestimmung in der Kategorienschrift weniger cindeutig formuliert ist als in den Untcrsuchungen der , Metaphysik“ kann hier außer Betracht bleiben. Für die spätantike und mittelalterliche Kommentierungstradition bilden Aristoteles' Schriften cin cinheitliches Corpus. Wenn Aristoteles irgendwo klare und überzcugende Definitionen gegeben hat, dann werden weniger prägnante Passagen im Licht dieser Definitionen interpretiert oder, wenn nötig, korrigiert. Aus einem ähnlichen Grund braucht uns auch hier dic Frage nicht zu berühren, ob die Kategorienschrift im ganzen und besonders ob deren letztc Kapitel autbentisch sind oder nicht. Es genügt, daß sic für die genannte Kommenticrungstradition als echt gelten.

59 In Cat., CAG 414,30-33 - CLCAG 569,12-15; vgl. CAG 415, 20-22 - CLCAG $570,38-40$. 
sam ist dann nicht dic nächste, sondern eine entferntc Gattung. Drittens gibt es ursprüngliche contraria, dic nicht mehr auf logisch frühere contraria zurückführbar sind, auf die dagegen anderc contraria als auf ihre Prinzipien zurückführbar sind $\$$.

Gegen diese Interpretation ist manches einzuwenden. Sie beseitigt das Anstößige allzu gewaltsam. An die Stelle von ,Gut und schlecht sind nicht in einer Gattung" tritt ,Gut und schlecht sind nicht in konträren Gattungen'; an die Stelle von „Gut und schlecht sind Gattungen für anderes“ tritt ,Gut und schlecht sind Prinzipien für anderes konträr Entgegengesetztes $^{661}$. Uberdies ist die Einteilung nicht homogen. $1 \mathrm{~m}$ ersten und zweitcn Glied werden konträre Arten bchandelt, im dritten aber eher konträrc Unterschicde. Würde man auch im ersten Fall statt der Arten dic artbildenden Diffcrcnzen einsetzen, so wärc nicht mehr zu sehen, worin sich der dritte Fall vom ersten noch unterscheidet ${ }^{62}$. Schließlich ist die Voraussetzung der Interpretation angreifbar, daß, gut' und ,schlecht' Qualitätsbestimmungen sind und in keiner anderen Kategoric ihren legitimen Platz haben.

Die Gegeninterpretation setzt gerade hier an. Nach ihr unterscheidet Aristoteles zwischen homonymen und nicht homonymen contraria, wobei dic nicht homonymen contraria nochmals in zwei Unterarten unterschieden werden $^{63}$. ,Gut" ist homonym im Sinne des „,von einem (Bezugspunkt) her" 64 gemäß, ,früher und spätcr" "65, ,von vielem "st, ,in mchrfachem Sinn" "67 Gesagtem; dasselbe gilt nach dieser Interpretation für den Gegenbegriff ,schlecht". Homonyma haben zwar einc ,gemeinsame Benennung", nicht aber eine gemeinsame Definition ${ }^{68}$. Sie können also auch nicht unter eincn gemeinsamon Gattungsbegriff fallen; denn sonst würde zumindest dieser von all ihren Instanzen univok prädiziert ${ }^{(19}$. Im Anschluß an Theophrast macht Simplikios darauf aufmerksam, daß es außer den contraria, gut schlecht" noch andere "Prinzipicn von Konträrem" gibt, die ihrerseits konträr und "nicht in einer Gattung" sind. Genannt werden die Gegensätze

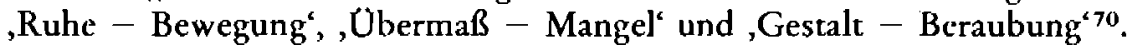

60 In Cat., CAG 415,9-13 - CLCAG 570,26- 30; CAG 415,22-28 -.. CLCAG 570, 40-46; CAG 416,3-7 - CI.CAG 571,57-62; vgl. CAG 416,8-20-CI.CAG 571, 63-76.

$61 \mathrm{Vgl}$. in Cat., CAG 415,9.13-14 CLCAG 570,26.30-31; CAG 415,32-33 CLCAG 571, 51 .

62 In Cat., CAG 415, 14- I5 - Cl.CAG 570,31-32.

63 In Cat, CAG 414,33-415,7 - CLCAG 569-570, Zeilen 15-24. Nach Simplikios' Auskunft stammt diese Interpretation von Phikosophen aus der Umgebung des Porphyrios.

64 In Cat., CAG 415,3-4 - CLCAG 570, 20-21.

b5 In Cat., CAG 415,30 -. CLCAG 570, 49.

6h In Cat., CAG 415,3 - CLCAG 570, 20

67 In Cat., CAG 415,22 - CI.C.AG 570,39.

6 Aristoteles, Cat. 1, 1 a 1-2.

69 Vgl. Simplicius, In Cat., CAG 415, 28-30 -- CLCAG 570,47 …49.

70 In Cat., CAG 415, 15-19- CLCAG 570, 32-37. 
Vorausgesetzt, daß auch diese Gegensätze nicht nur unter eine einzige Kategorie fallen, wäre auch auf sie die Interpretation im Sinne der Homonymie-Theorie auszudehnen.

Eine befriedigende Antwort auf dic aufgeworfenen Fragen gibt jedoch auch diese Interpretation nicht. Wenn Homonyma nicht unter einen gemeinsamen Gattungsbegriff fallen, dann können sie auch nicht Gattungsbegriffe sein. Aristoteles' Formulierung wäre irreführend; nach ihr müßten ,gut und, schlecht ${ }^{6}$ von ihren Instanzen univok prädiziert werden ${ }^{71}$. Außerdem bestätigt diese Interpretation den Konflikt mit der Begriffsbestimmung von Kontrarietät, statt ihn aufzulösen ${ }^{72}$.

Aristoteles scheint die Bestimmungen, gut' und ,schlecht ${ }^{\circ}$ bald als Unterscheidung von Haltungen, Verfaßthciten oder Beschaffenhciten der Kategorie des Beschaffenseins unterzuordnen, bald umgekehrt bestimmte Beschaffenheiten dem Guten und andere Beschaffenheiten dem Schlechten unterzuordnen ${ }^{73}$. Die Vereinbarkeit beider Ordnungen wird nicht gezeigt. Angesichts dieser Sachlage nehmen einige Interpreten ihre Zuflucht zu der Auskunft, Aristoteles spreche in der strittigen Passage gar nicht in eigenem Namen, sondern er passe sich der Auffassung der Pythagoräer an, deren ganze Theorie auf einer Lehre über einander entgegengesetzte Prinzipien beruht. Nach dieser Lehre repräsentiert in allen Gegensätzen je das,,rechte “ Glied das Bessere und das "linke" Glied das Schlechterc. Simplikios, außerstande, die Probleme, die er scharfsinnig aufgewiesen hat, zu lösen, schlicßt sich dicsen Interpreten vorsichtig an ${ }^{74}$.

\section{Nochmals: Der Gegensatz, gut - schlecht' als Gegensatz von Haben und Beraubung (Simplikios)}

Während sich dic bisher dargestellte Diskussion mit einem einzigen Satz aus der Kategorienschrift befaßte, der gewiß so, wic er da steht, nicht zu halten ist, wirft Simplikios im folgenden, auf Aristoteles' Ausführungen über die Gegensätze zurückblickend, ein grundsätzliches Problem auf. Er fragt, ob Aristoteles' Standardbeispielc für Konträrcs, nämlich ,das Gute das Schlechte', ,Trefflichkeit - Schlechrigkeit', ,Tüchtigkeit - Minderwertigkeit', ,Gerechtigkeit - Ungerechrigkeit' und ,Gesundheit - Krankheit', richtig gewählt sind oder ob sie nicht eher - wie, Sehkraft - Blindheit ${ }^{*}$ sämtlich Beispiele für den Gegensatz von Haben und Beraubung sind ${ }^{75}$.

\footnotetext{
71 In Cat., CAG 415,7-8 - CI.CAG 570,24-25.

72 In Cat., CAG 415,34-416,2 - CLCAG 571,52-57.

${ }^{73}$ In Cat., CAG 415,8-9 - Cl.CAG 570, 25-26; vgl. CAG 388,4-13- CLCAG 529, $57-67$.

74 In Cat., CAG 415,30-416,2 - CLCAG 570-571, Zeilen 49-57; vgl. CAG 418, 6-9- CLCAG 573, 32-35.

75 In Cat., CAG 416,21-28 - CLCAC 571-572, Zeilen 77-85.
} 
Dieser Fragestellung licgt eine Bestimmung von Kontrarietät zugrunde, die zwar so nirgends in der Kategorienschrift zu finden ist, die aber als schulmäßige Fassung der aristotelischen Lehre gelten kann, daß spezifizierende Unterschiede jeweils konträr zueinander sind: Contraria sind,,gleich stark“, "gleichen Ursprungs" und jeweils „,beide naturgemäß "76. In diesem Sinn sind z.B. weiß und schwarz oder warm und kalt konträr ${ }^{77}$; es handelt sich hier um Unterschiede, mit denen keine Bewertung verbunden ist. In den genannten Beispielen des Aristoteles dagegen ist je ein Glied des Gegensatzes naturgemäß, das andere aber widernatürlich ( $\pi \alpha \varrho \alpha ̀ ~ \varphi v ́ o เ v)^{78}$. Beim Schlechten, bei der Krankheit, auch bei der Ungerechtigkeit handelt es sich nach Simplikios' Auffassung um Fälle von Mißglücken, von Zufalls-

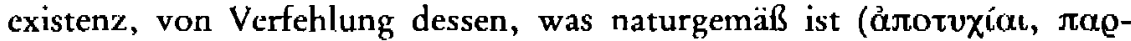

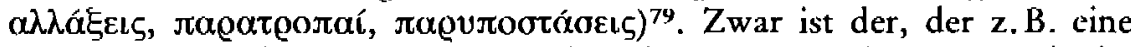
Augenkrankheit hat, der Sehkraft nicht vollständig beraubt wie der Blinde, aber er befindet sich doch in eincm Prozeß des der Sehkraft Beraubtwerdens ${ }^{80}$. Simplikios findet es so offensichtlich verfehlt, nur das vollständige Beraubtsein ,Beraubung zu nennen, das Beraubtwerden aber dem Konträren zuzuordnen ${ }^{81}$, daß er sich fragt, wie ein solcher Fehler übcrhaupt zustande kommen konnte. Als Erklärung bleibt dic Vermutung, daß Aristoteles und seine Nachfolger sich von der pythagoräischen Tradition nicht befrcicn konnten, „welche die beiden einander zugeordncten Ele-

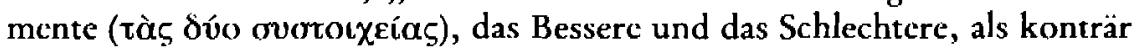
setzte ${ }^{\text {ct82 }}$.

In seiner zurückhaltenden Art deutet Simplikios die grundsätzliche Bedeutung, die er der vorgenommenen Korrektur beimißt, nur knapp an: Wenn man, "wo etwas widernatürlich ist, eher von ,Beraubung" als von ,Konträrem“" spricht, dann licgt darin auch dic richtige Antwort „gegen

76 In Cat., CAG 416,29-30 - CLCAG 572,85-87; CAG 416,32-33 -- CLCAG 572, 89-90 (ungenau übersetzt); CAG 417,8-9 - CLCAG 572,99-00; CAG 417, 27 - CLCAG 573,19-20; CAG 418,15-16- CICAG 574, 42-43.

77 In Cat., CAG 417,8-9 - CI.CAG 572,99 00.

78 In Cat., CAG 416,33-417,1 - CLCAG 572,90-91; CAG 417,4-7 - CLCAG 572,95-99; CAG 418,1-2 - CLCAG 573, 26-27; CAG 418,7 - CLCAG 574, 44.

79 In Cat., CAG 416,30--32 - CLCAG 572,87-89; CAG 417,1-4 - CI.CAG 572, 91-95; CAG 417, 27-29 - CLCAG 573, 20-22; CAG 418, 16-17 - CLCAG 574, 44-45. CAG 418,5-6 - CLCAG 573,31-32 wird Iamblichos als Hauptzcugc für die Auffassung

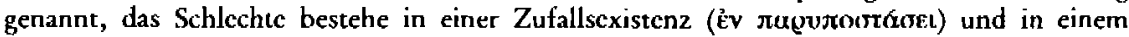

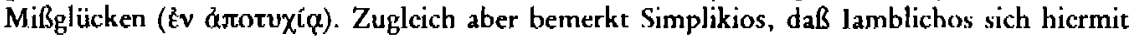
nicht gegen Aristotcles habe richten wollen; er hat zu Simplikios' Verwunderung offenbar "nicht verstanden", daß er mit dieser Theorie dic aristotclische Ansicht, ,gut" und, schlecht" seien konträr, aus den Angeln hebt (CAG 418,4-6 - CI.CAG 572, 29-32).

80 In Cat., CAG 417,9-29 - CLCAG 572-573, Zeilen 1-22.

81 In Cat., CAG 417,12-15 - CLCAG 572,3-7.

82 In Cat, CAG 418,3-9, ziticrt 418,7-8-CLCAG 573,28-35, zitiert 573, $32 \cdots 34$. 
die, die das Schlechte als Prinzip setzen" ${ }^{\text {"83. }}$. Man kann versuchen, in derselben Richtung weiterzudenken. Liegt nicht in dem Vorschlag, das Schlechte als Beraubung zu fassen, auch die richtige Antwort auf die im vorigen Abschnitt diskutierten Probleme? Die Thesen, daß (1) contraria das jeweils in einer Gattung am meisten Unterschiedene sind und daß (2) ,gut ${ }^{\star}$ und ,schlecht' überkategoriale und also homonyme (analoge) Prädikate sind, stehen nur dann in Konflikt miteinander, wenn der Gegensatz ,gut - schlecht' als konträrer Gegensatz gilt. Der Konflikt ist beseitigt, wenn die zweite These eine andere Gegensatzart, nämlich den Gegensatz. von Haben und Bcraubung, betrifft.

Allerdings hat auch Simplikios' Korrekturvorschlag einen Makel. Die glatte Konsistenz ist nicht ohne Preis erkauft. Geschwunden ist die ursprüngliche Phänomencinsicht, von der Aristoteles ausgegangen war: In der Ethik verwendet man die Bestimmungen ,gut' und ,schlecht', um Haltungen oder Einstellungen, aus denen heraus jemand handelt, zu spezifizieren. Schlechtigkeit wic auch besondere Fchlhaltungen wic Ungerechtigkeit oder Feigheit sind keine Weisen des zufälligen Mißglückens von Trefflichkeit bzw. von Gerechtigkeit oder Tapferkeit. Sofern über menschliches Handeln gesprochen wird, ist die Zuordnung des Gegensatzes, gut schlecht' zu dem Gegensatztyp ,Kontrarietät' zutreffender als die Zuordnung zum Gegensatztyp ,Haben - Beraubungs.

\section{Ungelöste Fragen}

Wenn man mit dem differenzierten Instrumentarium der aristotelischen Gegensatztheorie den Gegensatz ,gut - schlecht' untersucht, kommt man nicht um das Eingeständnis herum, daß das Schlechte dem Guten nicht stets in derselben Weise entgegengesetzt ist. Die diskutierten Verallgemeinerungen, sowohl die, ,gut " und ,schlecht' seien stets wie Haben und Bcraubung entgegengesetzt', als auch die, ,gut' und ,schlecht' seien typischerweise konträr, erweisen sich als kontraintuitiv. Gesucht ist eine Theorie, die genau bestimmt, wann, in welchen Bedcutungsformen und Verwendungsweisen, der Gegensatz von ,gut ' und ,schlecht' als Gegensatz von Haben und Beraubung und wann er als konträrer Gegensatz aufzufassen ist. In den aus den Kommentaren des Johannes Philoponos und des Simplikios zur Kategorienschrift referierten Passagen wird die Verschicdenheit dieser Gegensatzarten betont. Aristoteles hatte eher ihre Verwandtschaft, die „Rückführbarkeit“ der Kontrarietät auf den Gegensatz. von Haben und Beraubung, unterstrichen, ohnc jedoch das Verhältnis zwischen beiden Gegensatzarten ganz. klar begreifbar zu machen. Hier ist anzusetzen. Im Ausgang von der Bestimmung des Schlechten als Beraubung ist begründend

${ }^{83}$ In Cat., CAG 418,1-3 - CICCAG 573,26-28. 
zu erklären, unter welchen Bedingungen es angemessen ist, von der Entgegensetzung ,Haben - Beraubung zur Entgegensetzung ,Kontrarietät überzugehen, und was dieses Úbergehen genau bedeutet ${ }^{84}$.

\section{Thomas von Aquins Problemlösung}

Im folgenden soll nachgewiesen werden, daß sich aus Untersuchungen des Thomas von Aquin eine Theorie des Gegensatzes ,gut - schlecht* gewinnen läßt, die die dargestellten Probleme überzeugend löst. Diese Theorie tritt freilich bei Thomas nirgends in den Vordergrund. Die Analyse der logischen Struktur des Gegensatzes zwischen ,gut' und ,schlecht bildet nicht das Thema einer Abhandlung oder eines Artikels, sondern sie gehört zum Argumentationsinstrumentarium, mittels dessen theologische und philosophische Sachfragen präzisiert, geklärt und beantwortet werden. Auf diesen Argumentationshintergrund richten wir unsere Aufmerksamkeit, um die Konsistenz und Erschließungskraft von 'Thomas' Theorie des Guten und des Schlechten zu erweisen.

\section{,Gut' als transzendentales Prädikat}

Die 'T'ranszendentalienlehre als Grundlage dieser'Theorie muß als bekannt vorausgesetyt werden. Nur die für unser Vorhaben unerläßlichen Bestimmungen seien in möglichster Kürze crinnert ${ }^{85}$. ,Gut ${ }^{*}$ gehört zu den überkategorialen, mit , seiend' konvertiblen Bestimmungen. In ihnen wird etwas ausgedrückt, was in der Bezeichnung, seiend' nicht ausgedrückt ist. Die transzendentalen Prädikate fügen $\mathrm{zu}$,seiend" keinen neuen Sachgehalt hinzu; denn , seiend' umfaßt alle Sachgehalte. Sie bezeichnen auch keine besondere Seinsweise. Das durch sie zu ,sciend" Hinzugefügte ist eine begriffliche Artikulation cines Sinngchalts.

Der durch ,gut' zu ,sciend' hinzugefügte begriffliche Aspekt wird ausgelegt als Zielhaftigkeit.

„Der Sinngehalt von, gut' besteht darin, daß etwas geeignet ist, ein anderes zielhaft $\mathrm{zu}$ vollenden. " 86

H4 Beiläufig sei darauf hingewiesen, daß dic deutsche Sprache cincn ähnlichẹn Ansatz. nahelegt. Als "Ubel“ ist das Schlechte Beraubung des Guten, als „Büses“ konträrer Gegensatz zum Guten. Beide Verwendungsweisen sind aber nicht ohne Bezug zueinander: Das Böse ist selbst ein Úbel.

*5 Thomas von Aquin, De ver. q. 1 a. 1 ; q. 21 a. 1; vgl. S.th. I q. 5 a. 1.

H, De ver. q. 21 a.2: Cum ratio boni in boc consistat quod aliquid sit perfectivum alterius per modum finis, omne illud quod invenilur habere rationem finis babet etiam rationem boni. Vgl. q. 21 a. 1: Primo et principaliter dicitur bonum ens perfectivum alterius per modum finis; sed secundario dicitur aliquod bonum quod est ductivum in finem. 
In dieser Explikation des Sinngchalts von, gut' wird ein Verhältnis zwischen eincm vollendeten Ziel und einem ,anderen", das vollendbar ist, gesetzr. Diescs Verhältnis muß nicht ein Diskrepanzverhältnis sein. Im Gegenteil: Wenn ,gut' nur begriffliche Explikation von ,seiend' ist, nichts, was zu ,seiend" noch real hinzugefügt werden könnte, dann ist auch die Unterscheidung zwischen dem vollendenden Ziel und dem durch dieses Ziel vollendbaren „Anderen“" nur begrifflich. Sofern das "Andere“ ist, ruht es auch im Zic] und ist es auch gut ${ }^{87}$. Nun besagt ,Sein ' für Thomas ,Aktualität'; ihr gegenüber steht ,Potentialität'. Erst aus dieser Spannung z.wischen Potentialität und Aktualität resultiert die mögliche Diskrepanz zwischen dem Vollendbaren und sciner Vollendung. Finzig Gott, der rcine Aktualität ist, der nicht nur am Sein teilhat, sondern das Sein selbst ist, ist ganz gut ohne jede Möglichkeit der Beimischung eines Schlechten ${ }^{\mathrm{Bs}}$. Für alles Geschaffene gilt: Fs ist gut in dem Maße, in dem es aktual ist; es ist aber nicht "schlechthin gut", sofern ihm Potentialität beigemischt ist. Thomas nennt das Gutsein, das jedem Seienden zukommt, sein ,wesenhaftes Gutsein" (bonitas essentialis, bonitas substantialis); er betont, daß etwas gemäß diesem wesenhaften Gutsein nur ,in einer bestimmten Hinsicht" (secundum quid) ,gut" genannt werde. Zum Gutsein schlechthin würde gehören, daß etwas auch in seinen akzidentellen Bestimmungen vollendet ist ${ }^{89}$.

,Schlecht' ist in diesen metaphysischen Erörterungen überall nur der privative Gegenbegriff zu, gut'. Schlechtigkeit ist nichts anderes als mangelnde Vollendung ${ }^{90}$.

\section{Die einem Ding eigentümlich zukommende Vollendung - Scblechtigkeit als Mangel}

„Offenkundig ist nicht allen Dingen ein und dieselbe Vollendung eigen, sondern verschiedenen Dingen eine je verschiedene." 91 Wenn, gut t von einem bestimmten Seienden prädizierbar sein soll, dann muß in dic allgemeine Sinnbestimmung (ratio communis) von ,gut' eine Differenzierungsanweisung eingearbeitet werden, die anzeigt, daß das Prädikat, gut' im Verhältnis zu dem Subjekt, von dem es ausgesagt wird, bestimmt aufzufassen ist. Thomas spricht von der einem Ding (res) je ,eigentümlichen

${ }^{87}$ De ver. q. 21 a. 2: Ipsum . . . esse habet rationem boni, unde sicut impossibile est quod sit aliquod ens quod non habeat esse, ila necesse est ut omne ens sit bonum ex boc ipso quod esse habet.

${ }^{\text {sa }}$ Vgl. S.c.g. I cc. 37-41, besonders c. 39; De ver. q. 21 a. 5; De malo q. 1 a. 2.

89 De ver. q. 21 a. 5 , c.a. und ad 1 ; 5 .th. 1 q. 5 a. 1 ad 1.

$90 \mathrm{Vgl}$. De malo q. 1 aa. $1-2$; S.th. I q. 48 aa. 1-4; S.c.g. III c.7.

91 De malo q. 2 a. 4: Manifestum est autem quod non est eadem perfectio propria omnium, sed diversa diversorum. 
Vollendung" ${ }^{\text {992 }}$ oder von dem einem Ding „entsprechenden“ Gutsein ${ }^{93}$.

Durch diese Differenzierungsanwcisungen wird zum einen angezeigt, daß Dingen unterschicdlicher Art auch eine unterschiedliche Vollendung "wesenhaft" zukommt. Sie zeigen aber wciter auch an, daß wir durch das Prädikat ,gut ' bestimmte Individuen im Hinblick auf bestimmte Eigenschaften, Verhaltensweisen oder Tätigkeiten auszeichnen können. Wird ein bestimmtes Individuum , schlecht ' genannt, so ist damit gemeint, daß ihm etwas fehlt, was ihm ,von Natur zugehört und was es besitzen soll“994. Besonders im Hinblick auf Akte, Tätigkeiten oder Vollzüge gebraucht 'Thomas die Begriffe ,konvenient' und, inkonvenient'. Der Sinngehalt von ,gut' und ,schlecht' kann differenzicrend folgendermaßen expliziert werden:

Ein Akt ist in dem Maße gut, in dem cr ,,der Natur des Agierenden konvenient ist";

cr ist in dem Maße schlecht, in dem er der Natur des Agierenden nicht konvenient ist ${ }^{95}$.

Wcnn wir eine Verhaltensweise, eine Eigcnschaft oder einen Akt eines Tieres, gut ${ }^{\dagger}$ oder , schlecht $t^{*}$ nennen, dann ist - ausgeschlossen den Fall, in dem wir nur die Brauchbarkeit für unsere eigenen Zwecke im Auge haben - damit gemeint, daß die entsprechenden Organe in Ordnung oder nicht in Ordnung, gesund oder krank sind. Wir beziehen uns auf die spezifische Natur des Subjekts, von dem wir sprechen. Dic Prädikate, gut ${ }^{c}$ und ,schlccht' selbst haben keinen spezifizierenden Sinn"6. Der Gegensatz, ist der von Haben und Beraubung, nicht der der Kontrarietät.

\section{,Gut' und ,schlecht' als konträre Spezifikationen von Gegenständen menschlichen Handelns: Begriffsbestimmung}

In der Ethik jedoch sind nach Thomas, gut' und ,schlecht' als einander konträr aufzufassen. Wenn wir menschliche Verhaltens- oder Handlungs-

92 De malo q.1 a. 2: Quamvis autem qundcumque ens, sive in actu, sive in potentia, absolute bonum dici possit, non tamen ex hac ipso quaelibet res est bonum boc. . . Tunc dicitur aliquid bonum boc, quando habet perfectionem propriam.

$93 \mathrm{Vgl}$. z. B. De malo q. 2 a. 4.

94 Vgl. 7. B. S.c.g. III c.6, n. 1899: Malum quidem in substantia aliqua est ex eo quod deficit ci aliquid quod natum est et debet habere; c.7, n. 1911: Malum . . nibil est aliud quam privatio eius quod quis natus est et debet babere.

95 De malo q. 2 a. 4: In rebus autem naluralibus actus bonus est qui est secundum convenientiam naturat agentis, malum autem qui non convenit naturae agentis. Vgl. S. th. I-II q. 18 a. 1.

${ }_{96} \mathrm{Vgl}$. S. th. I-II q. 18 a. 1: De bono et malo in actionibus oportet loqui sicut de bono et malo in rebus: eo quod unaquaeque res talem actionem producit, qualis est ipsa. Im Argument 1 des Artikels 5 derselben quacstio wird dieser Satz als Obersatz mit folgendem Unter- 
weisen, gut' oder, schlecht' nennen, dann haben diese Prädikate spezifizierenden Sinn. Thomas ist sich klar darüber, daß er für diese These Argumente beibringen und starke Gegenargumente entkräften muß ${ }^{97}$. Er tut dies, indem er die Vernunft als Tätigkeitsprinzip von den Tätigkeitsprinzipien nicht-vernunftbegabter Wesen unterscheidet.

Im eigentlichen Sinn menschliche Handlungen (actiones proprie humanae) sind Handlungen, die aufgrund von Vernunfterwägungen gewollt $\operatorname{sind}^{98}$, deren Inhalt der Handelnde also aufgrund von Überlegung bestimmt. Uas, was eine Art von Handlungen von anderen Handlungsarten unterscheidet, nennt Thomas den „Gegenstand" (obiectum) der Handlung ${ }^{99}$, Obiectum ' ist in seinem Sprachgebrauch stets ein Relationsbegriff: Objekt ist ctwas in bezug auf cin Tätigkeitsprinzip ${ }^{100}$. Die Frage, ob eine Differenz im Objektbereich Handlungsarten spezifisch unterscheidet oder nur beiläufig ist, ist deshalb nur im Rückbezug auf das Tätigkeitsprinzip zu entscheiden ${ }^{101}$.

Die Frage nach dem Charakter der Differenz zwischen guten und schlechten menschlichen Handlungen läßt sich nun folgendermaßen formulieren: Läßt sich aus dem vernünftigen Wollen als Tätigkeitsprinzip eine Unterscheidung zwischen solchen Handlungsgegenständen, welche der Art nach gut sind, und solchen Handlungsgegenständen, welche der Art nach schlecht sind, gewinnen? Gibt es darüber hinaus eine Art von vernünftig gewollten Handlungsinhalten, so daß diese Inhalte weder gut noch schlecht im spezifischen Sinne sind ${ }^{102}$ ?

Der Handlungsgegenstand ist dasselbe wie das unmittelbar intendierte Handlungsziel. Daß Prozesse und Akte aus ihrem Ziel spezifiziert werden, gilt allgemein. Zumindest bei I ebewesen besteht die Antwort auf die Frage

satz verbunden: Sed in rebus bonum et malum non diversificant speciem: idem enim specie est bomo bonus et malus. Daraus wird gefolgert, daß ,gut' und ,schlecht' auch als Aktprädikate nicht spezifizieren. Diese liulgerung gilt für Thomas innerhalb der Naturphilosophic.

${ }^{97} \mathrm{Vgl}$. S. th. I-II q. 18 a. 5 arg. 2; De malo q. 2 a. 4 arg. 8, arg. 10.

98 S.th. I-Il q.1 a.1: Actionum quac ab homine aguntur, illae solac proprie dicuntur bumanae, quae sunt propriae bominis inguantum est homo. Differt autem homo ab aliis irrationalibus creaturis in boc, quod est suorum acturm dominus. Unde illae solae actiones vocantur propric humanae, quarum homo est dominus. Est autem homo dominus suorum actuum per rationem et voluntatem. . . Illae ergo actiones propric bumanae dicuntur, quae ex voluntate deliberata procedunt. Si quat autem aliae actiones bomini conveniant, possunt dict quidem bominis actiones; sed non proprie bumanac, cum non sint bominis inquantum est bomo. Vgl. q. 18 a. 5 ; De malo q. 2 a. 4 ; S.c.g. 111 c. 9.

ig Vgl. S.th. I -.II q. 18 a. 2 ; a. 5 .

Iou Vgl. hierzu besondcrs instruktiv die wissenschaftstheoretischen Quaestionen in Thomas' Kommentar In Boethium De Trinitate, bcsonders q. 5 a.1.

101 Eine bestimmte Objektdifferenz kann bezogen auf ein Tätigkeitsprinzip Akte spezifizieren, bezogen auf cin anderes Tätigkeitsprinzip aber beiläufig sein. $V_{g} l$. De malo q. 2 a. 4; S. th. I-II q. 18 a. 5.

$1132 \mathrm{Vgl}$. De malo q. 2 a.4; S. th. I- 11 q. 18 a.8. 
,Was ist das für einc Art von Prozeß? nicht in einer bloßen Beschreibung, sondern in der Angabe des Nächstzieles, dem dieser Prozeß dient ${ }^{103}$. Für den Menschen charakteristisch ist, daß er Ziele nicht nur erfaßt - dies tun auch Ticre, wenn sic auf Nahrung aus sind oder vor Bedrohung Schutz suchen -, sondern daß er sie als solche erfaßt. Fr verstcht, was ,Ziel' heißt (ratio finis), und dieses Verständnis ist die Bedingung dafür, daß er wollend seine Zicle bestimmen kann ${ }^{104}$. Wegen der aufgewiesenen engen Verbindung zwischen dem Ziclbegriff und der Explikation dessen, was ,gut' heißt, kann auch gesagt werden: Nur der Mensch versteht, was ,gut' heißt, und dieses Verständnis ist die Bedingung dafür, daß er entscheiden kann und muß, worein er - hier und jetzt oder auch langfristig - sein Gut zu sctzen wählt ${ }^{105}$. In jeder Handlung (und in jeder Unterlassung) wird hierüber entschieden. Die Frage, Was tust du? Was ist das für eine Handlung?" wird zwar zunächst inhaltlich durch Angabe des intendierten Ziels beantwortet. Wenn - was noch nicht gezeigt ist - sich aber Handlungen durch die Unterscheidungen , gut', ,schlecht', ,indifferent' spezifizicren lassen, dann ist dies die lezztlich relevante Spezifikation ${ }^{106}$.

Der Begriff der guten, der schlechten und der indifferenten Handlung ergibt sich, wenn man in die oben allgemein in bezug auf Akte eingcführten Explikationen als Tätigkeitsprinzip dic Vernunft einsetzt:

Eine menschliche Handlung ist der Art nach genau dann gut, wenn der Handlungsgegcnstand ctwas einschließt, was der Vcrnunft konvenient ist.

Eine menschliche Handlung ist der Art nach genau dann schlecht, wenn der Handlungsgegenstand etwas einschließt, was der Vernunft widcrstreitet.

Eine menschliche Handlung ist der Art nach genau dann indifferent, wenn der Handlungsgegenstand weder etwas einschließ was der Vernunft konvenient ist, noch etwas, was ihr widerstreitet $^{107}$.

Der Weg zum Erweis von Thomas' These, daß bezüglich menschlicher Handlungen ,gut' und ,schlecht' konträre, spezifizierende Bestimmungen sind, führt über den Nachweis der Erfüllbarkeit dieser Begriffe. Es wird

$103 \mathrm{Vgl}$. S. th. I-Il q. 1 aa. $2-3$.

104 S. th. I-II q. 1 a. 2.

105 Vgl. S. th. I-Il q. 1 a. 2 ad 3.

In Vgl. S. th. I-II q.1 a.3 ad 3.

107 Dc malo q.2 a.5: Est aucem aliquod obiectum quod importat aliquid conveniens rationi, et facit esse bonum ex genere, sicut vestire nudum; aliquod autem obiectum quod importat aliquid discordans a ratione, sicut tollere alienum, et hoc facit malum in genere; quoddam vero obiectum est quod neque importat aliquid conveniens rationi, neque aliquid a ratione discordans, sicut levare festucam de terra, vel aliquid buiusmodi; et buiusmodi dicitur indifferens. 
sich zeigen, daß für diesen Nachweis eine genaucre Erklärung des Kriteriums, der Vernunft konvenient' erforderlich ist.

Um Mißverständnisse auszuschließen, soll zuvor noch geklärt werden, was mit dem Begriff der der Art nach indifferenten Handlung genau gemeint ist. Nicht jedes Tun von Menschen ist eine im eigentlichen Sinn menschliche Handlung. Wenn jemand unüberlegt und unwillkürlich, ohne damit überhaupt etwas zu wollen, seine Hand oder seinen Fuß bewegt, so ist dies gewiß im allgemeinsten Sinn ein Tun dieses Menschen. Aber solches Tun ist $\mathrm{dem}$ von Thomas vorgeschlagenen Unterscheidungskriterium nicht zugänglich. Es liegt vor der Differenz zwischen ,der Art nach gut $^{\text {' }}$ und ,der Art nach schlecht ${ }^{\prime 106}$. Xhnliches gilt für das Handeln von Kindern. Die angegebenen Definitionen betreffen nur solche Handlungen, über die der Handelnde Herr ist ${ }^{109}$ und die ihm deshalb zugcrechnet werden können. Die Definition der der Art nach indifferenten Handlung ist also durch unwillkürliche oder unzurechenbare Handlungen nicht erfüllt; gefragt ist vielmehr, ob und in welchem Sinn cs cin menschliches, verantwortliches Handeln gibt, das in der Differenz von gut und schlecht steht, aber weder der Art nach gut noch der Art nach schlecht ist ${ }^{110}$.

\section{,Schlecht' als Spezifikation: Erfüllbarkeitsnachweis}

Da der Zwischenbereich des der Art nach Indifferenten nur negativ durch Abgrenzung gegen die Extrcme bestimmt ist, ist es logisch vorrangig, den Erfüllbarkeitsnachweis für diese Extrembegriffe zu crbringen. Problematisch scheint dieser Nachweis für dic der Art nach schlechten Handlungen. Wie kann es aufgrund von vernünftiger Úberlegung gewollte Handlungsgegenstände geben, die etwas cinschließen, was der Vernunft widerstrcitct?

Alles, was gewollt wird, wird notwendigerweise als ein Gutes (sub ratione boni) gewollt, und alles, was als ein Gutes aufgefaßt wird, ist auch in irgendeiner Hinsicht erstrebenswert, also gut ${ }^{111}$. Dächte man sich eine vollkommen isolierte Handlung, die keine weiteren Folgen für den Handelnden hätte und auch sonst niemanden beträfe, so gäbe es kcin Kriterium, sie als schlecht zu beurteilen. Aber gerade dieser Gedanke ist unmöglich; es handelt sich um cine Abstraktion, die das Phänomen des Handelns verfehlt. Dic Realisierung einer belicbigen Handlung impliziert zumindest,

$100 \mathrm{Vgl}$. S.th. I-II q. 1 a. 1 (ziticrt oben Anm. 98); ibid. arg. 3 und ad 3; De malo q. 2 a. 5 mit ad 6; S. th. I-II q. 18 a.9.

10\% Vgl. S.th. I-II q. 1 a. $1 ;$ q.6 a. 2 ad $2 ;$ q. 21 a. 2 .

$110 \mathrm{Vgl}$. S.th. I-II q. 18 a.5: Dicuntur. . aliqui actus humani, vel morales, secundum quod sunt a ratione.

111 Vgl. S.th. I-II q.8 a,1: Volunlas est appetitus quidam rationalis. Omnis autem appetitus non est nisi boni. Vgl. q. 19 a. 1 arg. 1 und ad 1; S.c.g. III cc. 3 -6. 
daß eine andere Handlung, die statt ihrer möglich gewesen wäre, unrealisiert bleibt. Dic unrealisierte Handlung aber hätte sich ebenfalls auf etwas gerichtet, was mindestens in irgendeiner Hinsicht ein Gutes ist, vielleicht ein Besseres. Aus der einfachen Tatsache, daß der Handlungswille je partikulär ist, folgt also, daß der Vernunftanteil an ihm nicht darauf beschränkt sein kann, nur etwas als gut vorzustellen, sondern eine Abwägung zwischen verschiedencn Handlungsobjekten impliziert. Die ausführliche Fassung der Definition der der Art nach schlechten Handlung lautet deshalb, daß ihr Handlungsgegenstand etwas einschlicßt, was der Ordnung der Vernunft widerstreitet ${ }^{112}$. Entsprechend sind die Definitionen für der Art nach gute und der Art nach indifferente Handlungen zu ergänzen.

Der Nachweis, daß ,schlecht' bezüglich menschlichen Handelns spezifische Differenz scin kann, besteht in folgenden Momenten: Eine schlechte Handlung ist möglich, sofern ihr Gegenstand in irgendeiner Hinsicht als gut vorgestellt wcrden kann. Sie ist schlecht, sofern der Gegenstand der Vernunftordnung widerstreitet. ,Schlecht' kann spezifische Differenz sein, sofern das Gewollte „etwas Positives“ ist, nämlich ein bestimmter Handlungsgegenstand ${ }^{113}$.

\section{Bemerkungen zur inhaltlichen Füllung des Begriffs, Vernunftordnung}

Auf die Art und Wcise, wie Thomas den ordo rationis inhaltich bestimmt, kann hier nur kurz eingegangen werden. Dic Beispiele, die Thomas konstant für spezifisch gute, spezifisch schlechte und spezifisch indifferente Handlungen verwendet, lauten; ,dem Bedürftigen Almosen geben“ oder „den Nackten bekleiden" für ,gut", „Fremdes wegnehmen" für ,schlecht", „aufs Feld gehen" oder derartiges für, indifferent"14. Diese Bcispiele zeigen, daß nach Thomas' Auffassung zur inhaltlichen Füllung des Begriffs der Vernunftordnung der Bezug auf den Mitmenschen gehört. Thomas rechtfertigt seinc Beispielwahl nicht ausführlich. Es genügt ihm, darauf

112 S.th. I-II q.18 a.8: Actus humanws, qui dicitur moralis, habet speciem ab obiecto relato ad principium actuum humanorum, quod est ratio. Unde si obiectum actus includat aliquid quod conveniat ordini rationis, erit actus bonus secundum skam speciem, sicut dare eleemosynam indigenti. Si autem includat aliquid quod repugnet ordini rationis, enit malus actus secundum speciem, sicut furari, quod est tollere aliena. Contingit autem quod obiectum actus non includit aliquid pertinens ad ordinem rationis, sicut levare festucam de terra, ire ad campum, et buiusmodi: et tales actus secundum speciem suam sunt indifferentes.

113 S.th. I-II q. 18 a. 5 ad 2: Dicitur. . malus actus secundum suam speciem, non ex eo quod nullum habeat obiectum sed quia babet obiectum non conveniens rationi, sicut tollere aliena. Unde inquantum obiectum est aliquid positive, potest constituere speciem mali actus. Vgl. De malo q. 1 a. 1 ad $12 ;$ q. 2 a. 4 ad 8 ; q. 2 a. 5 ad 3; S.c.g. III cc. 8-9.

114 Vgl. De malo q. 2 a. 5 (zitiert oben Anm. 107); S.th. I-Il q.18 a. 8 (zitiert oben Anm. 112). 
hinzuwcisen, daß die Vernunft, mit der dic gute Handlung übereinstimmt und mit der die schlechte Handlung in Widerstreit steht, die „durch das göttliche Gesetz cntweder auf natürliche Weise oder durch Belehrung oder durch gnadenhafte Eingießung geformte Vernunft" ist ${ }^{115}$. Man kann für Thomas' Beispielwahl auf der Grundlage des bercits erwähnten Nichtisolierbarkcits-Axioms argumentieren. Menschliches Handeln und Unterlassen betrifft nicht nur den Handelnden, sondern normalerweise auch andere, die wir ,seine Nächsten' nennen können. Wie wir durch eigenes Handeln das Wohlcrgehen anderer fördern oder becinträchtigen können, so sind wir auch in unserem eigenen Wohlergehen von Tun und Lassen anderer abhängig. Für dic Vernunftordnung, aufgrund dercr entscheidbar sein soll, welche Handlungsinhalte der Art nach gut, welche der Art nach schlecht und wclche der Art nach indifferent sind, ist das Faktum des sozialen Miteinanders konstitutives Moment. Dieses Faktum ist Rechtfertigungsgrund für die Maxime, bcim Abwägen möglicher Handlungsinhalte das Wohl des Nächsten zu berücksichtigen ${ }^{116}$. Fin Handeln, das ausdrücklich aus dieser Maxime entspringt, nennen wir, gut' oder zumindest ,gut gemeint'; cin Handeln, das ausdrücklich gegen diese Maxime verstößt, nennen wir ,schlecht ' oder ,böswillig'.

Thomas füllt den Begriff, Vernunftordnung' durch eine sehr differenzierte Darstellung der guten und schlechten menschlichen Grundhaltungen, der Tugenden und Laster, inhaltlich aus ${ }^{117}$. Dic Tugenden und die aus ihnen hervorgehenden Handlungen entsprechen der Vernunftnatur des Menschen; die Laster und die aus ihnen hervorgehenden Handlungen widerstreiten ihr ${ }^{118}$. Es muß hier genügen, die anthropologischen Positionen zu nennen, die Thomas' Abhandlungen über die einzelnen positiven und negativen Haltungen zugrunde liegen. Es ist der rationalen Natur des Menschen angemessen, die eigenen Tricbkräte (passiones) zu lenken und das erkannte Gute auch zu vollbringen; dem entsprechen die Tugenden des Maßes und der Tapferkeit. Es ist der sozialen Natur des Menschen angemessen, das Wohl seines Nächsten zu respektieren; dem entspricht die Tugend der

115 Dc malo q. 2 a.4: Loquimur autem nunc de actibus hominis: unde bonum et malum in actibus, secundum quod nunc loquimur, est accipiendum secundum id quod est proprium bominis in quantum est bomo. Haec autem est ratio, unde bonum et malum in actibus bumanis consideratur secundum quod actus concordat rationi informatae lege divina, vel naturaliter, vel per doctrinam, vel per infusionem.

116 Zum Versuch, den Begriff des Nächsten philosophisch zu adaptieren, vgl. G. H. von Wright, The Varieties of Goodness, I,ondon-New York 1963, X, $\$ 5 ; 1$. Craemer-Ruegenberg, Moralsprache und Moralität. Zu Thesen der Sprachanalytischen Ethik, Diskussion, Kritik, Gegenmodell, Frciburs-München 1975, VI, besonders 148-149; 156-160.

$117 \mathrm{Vgl}$. Dom O. Lottin, L'ordre moral et l'ordre logique d'après saint Thomas d'Aquin, in: Annales de l'Institut Supéricur de Philosophie 5 (1924) 301-399, besonders 323, 332-333, 389-392.

in Vgl. S. th. I-- II q. 54 a. 3. 
Gerechtigkeit, die bei Thomas nicht eng legalistisch gefaßt wird, sondern das gesamte Sozialverhalten des Menschen betrifft. Das allgemein als gut Erkannte muß konkret in situationsangemessene Finzelhandlungen umgesetzt werden; dem entspricht die Tugend der Klugheit. Es ist der kontingenten Natur des Mcnschen angemessen, anzucrkennen, daß er als Bild Gottes geschaffen ist; dem entsprechen die theologischen Tugenden des Glaubens, der Hoffnung und der Licbe.

\section{Beurteilung von Handlungsarten und Beurteilung von Einzelhandlungen}

Bisher wurde crörtert, welchc Arten von Handlungsinhalten allgemein als gut, wclche allgemein als schlecht und welche weder allgemeingülug als gut noch allgemeingültig als schlecht zu beurteilen sind. Bei der Beurteilung konkreter Einzelhandlungen kommen - außer dem Handlungsobjekt - weitere Momente zur Geltung: das Motiv des handelnden Individuums und dic Situation, in welcher die Handlung geschieht ${ }^{119}$. Wenn z. B. jemand Almosen gibt, nur weil er sich davon Reputation verspricht, wird man seine Handlungsweise nicht einfachhin ,gut' nennen. Das Urteil über einen Diebstahl wird sich zumindest mildern, wenn er begangen wird, um die Not eines Nächsten zu lindern ${ }^{20}$. Dieser letzterwähnte Fall kann andererseits auch als Beispiel dafür gelten, daß ein schlechtes Mittel einen guten Zweck korrumpicrt. Bei der Handlungsbcurteilung können ferner auch Umstände der Quantität oder des Ortes und der Zeit eine Rolle spielen. Fin Bankeinbruch wird anders beurteilt als ein Ladendiebstahl; der Kirchenraub gilt nicht einfach als Diebstahl, sondern als Sakrileg.

Dieser sehr weitgehenden Konkretisicrung steht aber eine Schwicrigkeit entgegen. Umstände sind Akzidentien der menschlichen Handlung ${ }^{121}$. Kennzcichnend für dic theoretische Philosophie ist - jedenfalls nach aristotelischer Konzeption, dic Thomas übernimmt - die strikte Scheidung zwischen Wesensbestimmtheit und akzidentellen Bestimmungen. Zwar sind auch die Naturdinge als Gegenstände der thcoretischen Philosophie konkret gegeben nur in ihrer akzidentellen Bestimmtheit; die Aufyabe der Theorie ist aber hier gerade, aus den individuellen Konkretionen die Wesenheit z.u abstrahieren ${ }^{122}$. Kann die Theorie der Praxis anders ver-

114 Der Betrachtung der Umstände widmet Thomas bercits in den Grundlegungsquacstionen der Moraltheologie eine ganze quaestis, S.th. I-II q.7. Vgl. besonders a.2, Utrum circumstantiae humanorum actum sint considerandac a tbeologo. Vgl. wcitcr S.th. I-II 4. 18 a. 3 ; De malo q. 2 aa.6-8.

$120 \mathrm{Vgl.} \mathrm{S.th.} \mathrm{I-II} \mathrm{q.} 18$ a. 4 arg. 3 und ad 3.

121 Vgl. S.th. I-II q. 7 a. 1 ; q. 18 a. 3 arg. $2 ;$ q. 88 a. 5.

122 Zur differenzicrteren Bestimmung der Abstraktionsarten vgl. dic wissenschaftsthcoretischen Quaestionen In Boethium De Trinitate, besonders q. 5 a. 3. 
fahren? Muß nicht auch sie gleichsam eine "lctzte Wesensform" fixieren und dic individuellen Besonderheiten aus der Betrachtung ausklammern? Wie ist es zu verstehen, daß eine aufgrund ihres Inhalts allgemeingültig als gut bestimmte Handlung im besonderen Fall wegen des subjektiven Tatmotivs nicht mchr gut, sondern schlecht sein soll? Wie ist es zu verstehen, daß eine bestimmte Art von Verfehlung wegen eines bloßen Situationsumstandes in eine andere Art von Verfehlung übergehen können soll?

Vor besonders augenfällige Probleme stellt 'Thomas' Auffassung von der indiffercnten Handlung. Thomas' These ist nämlich, daß jede spezifisch indifferente Handlung ,wegen irgendeines Umstands" doch entweder gut oder schlecht ist ${ }^{123}$, so daß es also eine individuelle Handlung, die indifferent ist, nicht gibt. Verwickelt sich Thomas hier nicht in einen Widerspruch, sofern er eine Art von Handlung behauptet, die kein Individuum enthält, also uncrfüllbar ist ${ }^{124}$ ?

Thomas verteidigt die These, daß „ein Umstand eine Spezies des guten und schlechten Aktes konstituieren kann" ${ }^{125}$. Das Theoriemodell der naturphilosophischen Wesensbetrachtung ist nicht auf dic praktische Philosophic übcrtragbar. Menschliche Handlungen sind anders konstituiert als Naturdinge. Während die Vernunft hinsichtlich der Naturdinge deren metaphysische Konstitution und spezifische Bestimmtheit lediglich abstrahicrend erfaßt, hat sic dic Konstitution und Bestimmung ihrer eigenen Akte selbst zu vollbringen.

„Wie die Arten der Naturdinge aus natürlichen Formen konstituiert sind, so werden die moralischen Handlungen aus Formen, sofern sic von der Vernunft (selbst) konzipiert sind, konstituiert. .. . Weil aber die Natur auf eines determinicrt ist und weil der Naturprozeß nicht ins Unendliche gehen kann, gelangt man notwendig zu ciner letzten Form, aus der die spezifische Differenz zu entnehmen ist, nach welcher es cinc wcitcre spezifische Differenz nicht geben kann. Und daher kommt es, $\mathrm{da} ß$ in den Naturdingen das, was Akzidenz. für eine Sache ist, nicht als Differenz, welche eine Art konstituiert, aufgefaßt werden kann. Der Fortgang der Vernunft dagegen ist nicht auf eines determinicrt, sondern die Vernunft kann über jede Gegebenheit stets weiter fortschrciten. Daher kann das, was bezüglich eines Aktes als ein Umstand aufgefaßt wird, welcher dem die Art des Aktes bestimmenden Gegenstand (nur) hinzugefügt ist, von der ordnenden Vernunft selbst wieder als relevante Bestimmung (principalis conditio) des die Art des Aktes bestimmenden Gegenstandes aufgefaßt werden." ${ }^{120}$

${ }_{12.3} \mathrm{~S}$, th. I-II q.18 a.9: Oportet quod quilibet individualis actus habeat aliquam circumstantiam per quam trahatur ad bonum vel malum. De malo q. 2 a. 5: Si . . loquamur de actu morali secundum individuum, sic quilibet particularis actus moralis neccsse est quod sit bonus vel malus propter aliquam circumstantiam.

$124 \mathrm{Vgl}$. S. th. I-II q. 18 a.9 arg. 1.

125 S.th. I-II q. 18 a. 10: Utrum aliqua circumstantia constituat actum moralem in specie boni vel mali.

126 S.th. I II q. 18 a. 10. 
Genau dies ist z. B. der Fall, wenn ein Diebstahl durch den Ort, an dem cr stattfindet, als Sakrileg beurteilt wird.

„Wann immer (also) cin Umstand cine(n) spezielle(n) (Aspekt der) Vcrnunftordnung betrifft, sei $e s$, daß er ihr gemäß ist, sei es, daß er ihr widerstrcitet, muß dicser Umstand dem guten oder schlechten moralischen Akt eine (ncue) Spezies geben. “127

Der Ubergang von der Beurteilung eincr Handlung aus dem, was im Handlungsobjekt der Vernunftordnung konvenient oder inkonvenient ist, zur Einbeziehung der jeweiligen Handlungsumstände und des persönlichen Motivs des Handelnden ist kein Ubergang zu einer neuen Art von Betrachtung ${ }^{12 *}$, sondern ein Ubergang von der Beurteilung abstrakter Typen von Handlungen zur Betrachtung von Handlungen, wic sie tatsächlich geschchen. Die Bestimmung der Handlung aus dem Objekt ist nur cin erster Schritt, der offen ist für weitere Spczifizierungen. Das Kriterium, nach dem menschliche Akte beurtcilt werden, blcibt dasselbe: Wic der Handlungsgegenstand, so werden auch die Umstände und die persönlichen Tatmotive daraufhin untersucht, ob sie der Vernunftordnung entsprechen oder widerstreiten. Für diesen Spezifikationsprozeß gibt es keine prinzipiclle Grenze, da dic Vernunft ihr Kriterium immer wieder anlegen kann. Damit ist nicht gesagt, daß bei der Beurteilung konkreter Handlungen jeder Umstand berücksichtigt werden müßte. Moralisch relevant sind nur solche Umstände, welche dem genannten Bcurteilungskriterium zugänglich sind ${ }^{29}$. Wohl aber kann gesagt werden, daß die Fntscheidung, welche Umstände moralisch relevant sind und welche nicht, nur konkret durch Analyse der jeweiligen Einzelhandlung zu treffen ist. Es ist für keinen Umstand von vornherein auszuschließen, daß er für das moralische Urteil ins Gewicht fällt.

Mit der zuletzt gemachten Bemerkung ist bereits angedeutet, warum es lcgitim und sogar notwendig ist, zwischen der crsten Bestimmung der Handlung aus ihrem allgemeinen Inhalt einerscits und deren Wciterbestimmung aus den individucllen Tatumständen und -motiven andererseits zu unterscheiden, obwohl doch dic Umstände und Motive streng genommen

127 S.th. I-II q.18 a. 10: Quandocumque aliqua circumstantia respicit specialem ordinem rationis vel pro vel contra, oportet quod circumstantia det speciem actui morali vel bono vel malo. Vgl. q. 18 a.5 ad 4. Thomas' Analyse des Fortgangs der ihre Gegenstände konstituierenden Vernunft ist von großer Tragweite. Sie ist gecignet, auch den grundsätzlichen Ansatz der neuzeitlichen experimentellen Naturwissenschaft zu beleuchten. Sofcrn nämlich "die Vernunft .. . die Natur (nötigt), auf ihre Fragen zu antworten“" (I. Kant, Kritik der reinen Vernunft, Vorrede zur zweiten Auflage, B XIV), konstituiert sie selbst ihrc Gegenstände; folgcrichtig kann auch diescr Bestimmungsprozeß keine Grenze haben, vielmchr kann das, was in einer Untersuchung als irrelevanter Umstand ausgeklammert wird, stets in einer weiteren Untersuchung als relevante Bedingung aufgefaßt werden.

$128 \mathrm{Vgl}$. S.th. $1-11 \mathrm{q} .18$ a. 7.

129 Vgl. De malo $q .2$ a.6 mit ad 2; q. 2 a.7; S. th. I-II q. 18 a. 3 ad $2 ;$ a. 5 ad 4. 
zur vollen Bestimmung des Handlungsobjekts gehören ${ }^{130}$. Die allgemeinen Urteile über Gutsein oder Schlechtsein von Handlungstypen beanspruchen, aus der Natur des Menschen als eines unter Mitmenschen handelnden Vernunftwesens verbindlich begründbar zu sein; dieser Anspruch ist argumentativ zu rechtfertigen. Urteile über singuläre Handlungen dagegen sind in dem Maße, in dem sie die jeweiligen kontingenten Handlungsumstände berücksichtigen, Ermessensurteile. Vollends gibt es für Urteile, was für persönliche Handlungsmotive im Spicl sind, kaum überprüfbare Argumente; solche Urteile beruhen auf Einfühlung und Intuition, und häufig genug kann nicht einmal der Handelnde selbst zuverlässig über seinc Motive Auskunft geben. Die Theorie über gutes und schlechtes Handeln bleibt als Theorie notwendig abstrakt. Diese Abstraktheit ist so lange unschädlich, wie sic deutlich gesehen wird. Thomas reflektiert sie als Kluft $z$ wischen allgemeiner Theorie und konkreter Handlung, indem er bereits in den Grundlegungspartien seiner Morallehre eindringlich darauf hinweist, daß die Umstände häufig und dic Motive stets für das Gutsein oder Schlechtsein des Handelns relevant sind, indem cr jedoch andererseits vermeidet, die Entscheidung über den Einzelfall vorweg durch eine Typologie möglicher Umstände und Motive inhaltlich zu reglementieren. Dic Situationsbcurteilung ist Sache der Klughcit als praktischer Tugend.

\section{Der Gegensatz zwischen, gut' und ,scblecht" bexüglich einzelner Handlungen}

Vergleicht man die allgemeinen Urteile über Handlungsarten mit den konkreten Urteilen über Einzelhandlungen, so fällt eine eigentümliche Asymmetrie der Werturteile auf. Fine konkrete Einzclhandlung ist nicht schon dann gut, wenn sie dem durch den Handlungsgegenstand bestimmten allgerneinen Typus nach gut ist, sondern erst dann, wenn geschieht, was soll, ,, wann es soll, . . . wie es soll und so weiter" 131 und wenn die Handlung

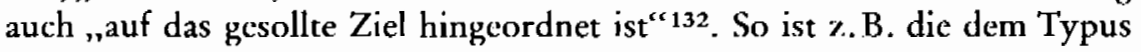

$130 \mathrm{Zu}$ den folgenden Oberlegungen vgl. die Reflexionen von Th. Gilby in seiner mit Anmerkungen und interpretierenden Appendices versehenen Obersetzung der Quacstionen 18-21 der I-Ilae: St. Thomas Aquinas, Summa theologiae, Vol, 18: Principles of Morality, London-New York 1966, besonders App. $1 \$ \$ 6-7$; App. 8, $\$ 1$; App. 9, $\$ 4$; App. 10; $\Lambda_{\text {pp. }} 11$; App. 12, $\$ 2$; ferner die Anmerkungen p. 8 und p. 15.

${ }^{131}$ De malo q. 2 a. 5: Non . . potest contingere quod actus singularis sine circumstantits fiat, quac ipsum recum faciant, vel indirecium. Si enim fiat quodcumque quando oportet et ubi oportet et sicul oportet etc., huinsmodi attus est ordinatus et bonus; si vero aliquid borum deficiat, actus est inordinatus et malus.

${ }_{132}$ S. th. 1 II q. 18 a.9: Cum . . rationis sil ordinare, actus a ratione deliberativa procedens, si non sit ad debitum finem ordinatus, ex boc ipso repugnat rationi, et babet rationem mali. Si vero ordinctur ad debitum finem, convenil cum ordine rationis: unde babet rationem boni. Necesse est autem quod vel ordinetur, vel non ordinetur ad debitum finem. Unde 
nach gute Handlung, einem Bedürftigen zu helfen, konkret erst dann einfachhin gut, wenn dem Bedürftigen das gegeben wird, was er wirklich braucht, auf eine Weise, dic ihn nicht demütigt, und wenn das Motiv der Handlung uneigennützig ist. Uneingeschränkt gut ist einc Einzelhandlung also dann und nur dann, wenn sie in jeder relcvanten Hinsicht, dem Objekt, den Umständen und dem Ziel nach, dem ordo rationis konvenient ist. Ist die Handlung in irgendeiner relcvanten Hinsicht dem ordo rationis inkonvenient, so ist sie in ebendicser Hinsicht nicht gut, sondern schlecht. Quilibet singularis defectus causat malum, bonum autem causatur ex integra causa lautet das von Thomas immer wieder zitierte Axiom des PseudoDionysius ${ }^{133}$. Aus dem Gesagten folgt: Das Verhältnis zwischen cinem abstrakt-allgemeinen und cinem konkrcten Mißbilligungsurteil ist von anderer logischer Struktur als das Verhältnis zwischen einem allgemeinen und einem konkreten Billigungsurteil. Die dem allgemeinen Typus nach gute Handlung kann im Einzelfall wegen irgendwelcher für das Werturteil relevanten Umstände oder wegen ihrer Zielsetzung schlecht sein. Die dem allgemeinen Typus nach schlechte Handlung aber kann in concreto nicht gut $\operatorname{sein}^{134}$; das Urteil über sic kann sich durch Einbeziehung von Umständen und Motiven nur mildern. Auch ehrenhafte Motive machen aus einer der Art nach schlechten, weil dem ordo rationis widerstreitenden, keine im Einzelfall gute Handlung.

Gibt es einerseits zwar Handlungsarten, die unter allen Umständen schlecht bleiben, nicht aber Handlungsarten, die unter allen Umständen gut bleiben, so gibt cs andererseits zwar Einzelhandlungen, die in jeder Hinsicht gut sind, nicht aber Einzelhandlungen, die in jeder Hinsicht schlecht sind ${ }^{135}$. Ein Rest von Vernunftkonvenienz. bleibt in jeder Handlung bestehen. Das Handlungssubjekt kann sich in seinem Streben und Wollen niemals vollkommen täuschen und niemals gänzlich pervertieren: Was immer gewollt wird, wird als ein Gutes gewollt, und auch das scheinbar Gute ist stets in irgendeiner Hinsicht wirklich erstrebenswert, also gut.

Die aufgewiesenen Asymmetrien der Werturteilc über Einzelhandlungen folgen schlüssig aus dem von Thomas zugrundegelegten Bewertungskriterium. , In jeder Hinsicht der Vernunftordnung entsprechend' ist ein Vollkommenheitsbegriff, dem gegenüber jede Einschränkung cinen Mangel bedeutet.

necesse est omnem actum bominis a deliberativa ratione procedentem, in individuo consideratum, bonum esse vel malum.

13.3 Ps.-Dionysius, De divinis nominibus, c. $4 \$ 30$; $\mathrm{gl}$. I'homas von Aquin, In De div. nom., L. XXII; vgl. die Litate in: S. th. $1-\mathrm{II}$ q. 18 a. 4 ad 3 ; 1)e malo $q .2$ a. 4 ad 2.

1.34 De malo q.2 a.4 ad 2: Actus malus non potest bene fieri; ex quo enim actus malus est, non potest esse integrum bonum; sed actus bonus potest male fieri, quia non requiritur quod sit integrum malum, sed sufficil quod sit particulariter malum. Vgl. 5.th. 1-II q.18 2.5 ad 4 .

1.35 Vgl. S.th. 1-11 q. 18 a. 8 ad 1. 
Diese Uberlegungen zeigen: Bezogen auf Einzelhandlungen gibt es kein Mittleres und kcinen Bereich der Indifferenz zwischen gut und schlecht. ,Einfachhin gut' und ,in irgendeiner Hinsicht schlecht' stehen sich als unmittelbare Gegensätze gegenüber ${ }^{136}$.

Moralisch neutrale Einzelhandlungen kann es nicht geben. Wenn nicht aufgrund äßerer Umstände, so wird doch jedenfalls aufgrund der zu jeder überlegt gewollten Handlung gehörigen Intention auf ein Ziel hin notwendig jede Finzelhandlung ,entweder zum Guten oder zum Schlechten hingezogen" ${ }^{137}$. Denn der Handlungsabsicht nach ist eine jede Handlung entweder auf das "gesollte Zicl" hingeordnet und damit der Vernunftordnung gemäß oder nicht; einen $Z$ wischenbereich, in welchem die Vernunftordnung weder positiv noch negativ tangiert wäre, kann es konkret nicht geben.

\section{Zwei disputierte Fragen in der Ethik - Hinweise auf ibre Beantwortung aus der vorgetragenen Analyse}

Man könnte die vorgelegte Analyse noch ergänzen, ctwa indem man zwischen spezifizierenden Umständen einerseits und das Gutsein odcr Schlechtsein einer Handlung nur vermehrenden oder vermindernden Umständen andererseits unterscheidet ${ }^{138}$ oder indem man das Verhältnis zwischen der Gutheit oder Schlechtheit der inneren Willensakte und der Gutheit oder Schlechtheit der äußeren Akte untersucht ${ }^{139}$. Ich will solche weiteren Differenzierungen und Präzisierungen hier nicht mehr vornehmen.

Es scheint mir jedoch lohnend, wenigstens kurz zu zeigen, wie man Thomas' Definitionen und Distinktionen gebrauchen kann, um umstrittene Fragen der Ethik und der Handlungtheorie zu klären. Ich wähle zwei Fragen aus, dic Thomas selbst - jedenfalls in der Form, in der ich sie formuliere - nicht untersucht hat.

Ein zentrales Problem der Ethik ist die Frage des Widerstreits zwischen mehrcren Handlungsregeln oder -normen, deren jede als gültig anerkannt ist. Im Zusammenhang unserer Untersuchung stellt sich das Problem des Normenkonflikts als Einwand gegen dic These, die der Art nach schlechte Handlung bleibe unter allen Umständen schlecht. ,Fremdes wegnehmen“

136. De malo q. 2 a. 5: Sic crgo bonus actus et malus actus ex genere sunt opposita mediala: et est aliquis actus qui in specie consideratus est indifferens. Bonum autem et malum ex circumstantia sunt immediata, qui distingusntur secundum oppositionem affirmationis et negationis, scilicet per boc quod est secundum quod oportet et non secundurn quod oportet secundum omnes circumstantias. Hoc autem bonum et malum est proprium actus singularis: et ideo nullus actus humanus singularis est indifferens.

137 S. th. I - II q. 18 a. 9 (zitiert oben Anm. 123 und $\Lambda$ nm. 132).

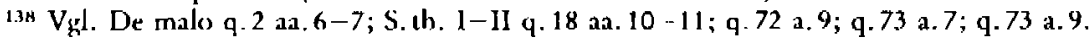

13.) Vgl. S. th. I-III q9, 19-20. 
wurde als Beispiel für eine der Art nach schlechte Handlung genannt. Ist diese Handlung aber ausnahmslos schlecht? Kann sie nicht unter Umständen sogar geboten sein, wenn z. B. das vom Ubberfluß des einen Weggenommene zur Linderung vitaler Not anderer dienen soll? Auf solche Fragen ist folgendermaßen zu argumentieren: Regeln wie ,Fremdes wegnehmen ist schlecht' sind Kurzformeln. Allgemeingültig und allgemcinverbindlich sind solche Regeln nur in Verbindung mit dem allgemeinen Kriterium: ,Fremdes wegnehmen ist schlecht, weil und sofern der Vernunftordnung widerstreitend'. Die Begründung stellt dic Geltungsbedingung der Regel dar. $\mathrm{Zu}$ prüfen ist, ob im konkreten Fall wirklich eine Verletzung der so begründeten Regel vorliegt. Für den im Beispiel genannten Fall heißt das: Zu prüfen ist, ob der Besitzer überhaupt cin aus der Vernunftordnung begründetes Recht auf das weggenommene Eigentum hat, ob also das Weggenommene wirklich ein „Fremdes“ ist. Falls die Antwort negativ ist, ist weiter zu prüfen, wer im konkreten Fall das Recht hat, das weggenommene NichtEigenc zu vergemeinschaften. Geprüft wird nicht, ob und unter welchen Umständen eine allgemeine Regel Ausnahmen zuläßt. Geprüft wird vielmehr, wie ein Tatbestand den Regeln zuzuordnen ist. In dieser Prüfung werden die Regeln selbst an ihrem Verbindlichkeitskriterium gemessen.

Die Frage, wie ,gut' und, schlecht' als Handlungsprädikate cinander entgegengesetzt sind, ist ein Thema auch der modernen Ethik, Metacthik und Handlungstheorie. Zur Beantwortung dieser Frage konkurrieren zwei Modelle. Die Disputanten orientieren sich entweder an der Logik des Sollens („deontische“", ,deontologische I,ogik“, ,Normenlogik“) oder an der Logik des Vorziehens (,prohairetische Logik“). Die erste Theorie gelangt zu folgender Klassifikation, dic analog zum logischen Quadrat darstellbar ist: Eine Handlung ist entweder allgemeingültig gut - d.h. geboten - oder allgemeingültig schlecht $-d$.h. verboten - oder moralisch neutral - d.h. freigestellt. ,Allgemeingültig gut' und ,allgemeingültig schlecht' sind entander konträr. Nach der zweiten Theorie wird hinsichtlich jeder Handlung entweder das Tun dem Unterlassen oder das Unterlassen dem Tun vorgezogen, d.h. entweder das Tun oder das Unterlassen wird gutgeheißen. Einen Zwischenbereich der Gleichgültigkeit oder Neutralität gibt es nicht. Die Aufgabe, beide Ansätze miteinander zu vermittcln, indem das relative Recht beider gezeigt wird, ist, wenn ich recht sehe, ungelöst. Die Vermittlung ist aber nötig. Einerseits kann die ethische Reflexion nicht hinnehmen, daß eine Klasse von Handlungen ins individuelle Belieben freigestellt bleibt. Gerade dann, wenn Tun oder Unterlassen nicht gesetzlich geregelt ist, stellt sich die Fragc, was denn hier ethisch richtig und was ethisch falsch ist. Andererseits blcibt die an der Logik des Vorziehens orientierte Handlungstheorie rein deskriptiv, wenn sie keine allgemeinen Kriterien erarbeitet, aufgrund derer begründet werden kann, warum das Tun einer bestimmten Handlung dem Unterlassen oder das Unterlassen dem Tun vorzuziehen ist. Thomas zeigt, wie beide Ansätze zueinander ins Verhältnis zu setzen sind. 
Der erste entspricht der Beurteilung von Handlungstypen, der zweite der Beurteilung von Einzelhandlungen. Dic Bcurteilung von Handlungstypen erstarrt bei Thomas deshalb nicht zu einer legalistischen Normentheorie, wcil Thomas die Notwendigkeit betont, diesc abstrakte Beurteilung in der Situation klug zu konkretisieren. Klugheit ist keine bloße Geschicklichkeit zu beliebigen Zwecken. Der Kluge, Einsichtige beurteilt seine Handlungssituation im Lichte von sittlich guten Vorentscheidungen, die Thomas (mit Aristoteles) ,ethische Tugenden' nennt.

„Man kann keine Klugheit haben, ohne die ethischen Tugenden zu haben. Denn Klugheit ist rechte Vernunft betreffs dessen, was getan werden kann. Sie geht - wie von Prinzipien - von den Zielen dessen, was getan werden kann, aus. Zu diesen aber verhält man sich durch die ethischen Tugenden in der rechten Weise. ${ }^{~}{ }^{140}$, , $\mathrm{Zu}$ einer guten Wahl gehört zweierlei. Frstens, daß man die gesollte Absicht auf ein Ziel hat; dies geschieht durch dic cthische Tugend, durch die das Streben zù dem der Vernunft entsprechenden Gut neigt, welches Gut das gesollte Ziel ist. Zweitens, daß man recht auffaßt, was zum Ziel führt; dies kann nur durch die recht beratendc, beurteilende und vorschreibende Vernunft geschehen; es gehört zur Klugheit ... Also kann es keine ethische Tugend ohne Klugheit geben." 141

140) S.th. I-II q.65 a.1; vgl. q. 58 a. 5; De virt, card. q. un. a. 2; Quodl. 12 q.15 a.1.

I+I S. th. I-II q. 58 a. 4; vgl. De virt. card. q. un. a. 2; Quodl. 12 q. 15 a. 1. 